\title{
The Preparation of Polypyrrole / Cellulose Acetate Composite Films and their Electrical Properties
}

\author{
Longning Suo ${ }^{a}$, Xiuli Shang ${ }^{b}$, Rongping Tang ${ }^{c}$, Yanqing Zhou ${ }^{d}$ \\ Department of Petrochemical Engineering, Lanzhou Petrochemical College of Vocational \\ Technology, Lanzhou 730060, China \\ asuolongning@163.com, ${ }^{b}$ Izshsxl@163.com, ctangzihan@163.com, ${ }^{\mathrm{d}}$ zhouyanqing2005@126.com
}

Keywords: Composite films; Polypyrrole/Cellulose acetate; Preparation; Resistance.

Abstract. A new method was developed for directly preparing conductive polypyrrole (PPy)/cellulose acetate (CA) composite films by in-situ chemical polymerization. The surface morphology of the films was observed by the atomic force microscopy (AFM). It was found that the composite films were composed of two layers with different structures. FTIR spectroscopy was used to analyze the chemical components of each layer. The thermo-oxidative degradation of the films was studied by thermogravimetric analysis (TGA). The results indicated that the thermal stability of the PPy/CA film is higher than that of the pure CA film.

\section{Introduction}

In recent years, conducting polymers with conjugated double bonds have attracted much attention as advanced materials. Among these conducting polymers, polypyrrole( PPy) is one of the most extensively studied polymers because of its high electrical conductivity and novel physicochemical property. These properties are favorable to its applications in the electrodes of rechargeable batteries or fuel cells, and electroluminescent diodes[1-5], sensors [6,7]and electrocatalytic materials [8].

It is generally known that PPy prepared directly by chemical or electrochemical polymerization is insoluble and infusible [9]. Its poor processibility of PPy impedes further investigation of its characteristics and limits its practical application. To overcome this disadvantage, several attempts have been made to prepare blends or composite materials containing PPy [10]. This article presents a new method for preparing PPy/CA composite film with a typical asymmtric structure by phase separation and in-situ polymerization. In comparison with the method described in the literature [11,12]. One of its advantages is that the film formation and the oxidation polymerization of pyrrole monomer occur simultaneously, which makes the conductive layer of the composite films thin, dense and high conductive, furthermore, adhered to the cellulose acetate substrates tightly. The chemical components and the surface morphology of the composite film were analyzed by using FTIR and observed by AFM technique, respectively. In addition, the influence of experimental conditions on the resistance was investigated in details.

\section{Experimental}

Pyrrole was purified by distillation and stored in refrigerator at about $4^{\circ} \mathrm{C}$. All other chemicals used were of analytical grade and used as received.

Cellulose acetate (CA) was dissolved in organic solvents at room temperature. Pyrrole monomer was slowly added to the solution containing CA. The mixture was coated directly on glass supports and then immersed in $\mathrm{FeCl}_{3}$ aqueous medium. After 30 minutes the film was peeled off the glass supports. Finally, the conductive films were dragged from the vessel using forceps, rinsed with deionzed water and dried in the air.

FTIR measurements of the films were performed in an ATR model on Nicolet NEXUS 670 spectrometer. The AFM images of the composite films was obtained with SPI3800-N(Seiko Instruments Inc). Thermogravimetric analysis was performed on the Shimadzu TA-50WSITG and DTA instruments with a heating rate of $10^{\circ} \mathrm{C} / \mathrm{min}$ in air. 


\section{Results and discussion}

The morphological features of PPy /CA films fabricated by the atomic force microscope (AFM). As shown in Fig. 1, the CA layer had a very smooth surface with pores, whereas the layer of PPy was relatively rougher. A number of clusters composed of polypyrrole chains, which grew on cellulose acetate substrates and seemed to be perpendicular to the film surface, were clearly seen from Fig 1b. In addition, some pores were distributed among the clusters of vertical polypyrrole chains.
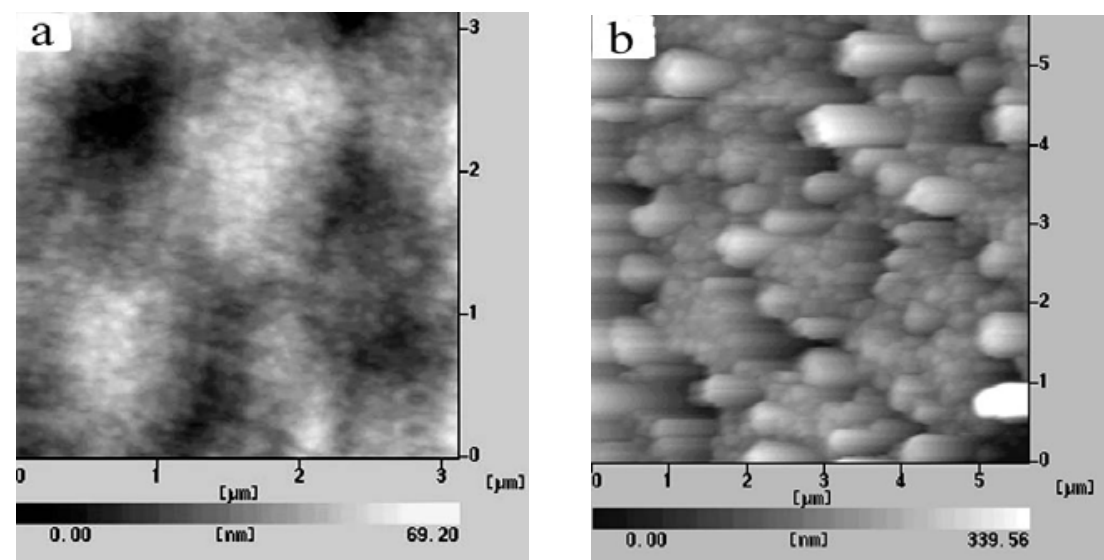

Fig. 1 AFM two-dimensional micrographs of (a) pure CA films, and (b) PPy-CA films.

FTIR spectra of pure PPy, PPy layer of composite films and pure CA were shown in Fig. 2, respectively. For pure PPy (a), the peaks at 1558 and $1477 \mathrm{~cm}^{-1}$ were assigned to $\mathrm{C}=\mathrm{C}$ stretching vibrations and $1302 \mathrm{~cm}^{-1}$ was deformation vibration. The absorption frequency at $1192 \mathrm{~cm}^{-1}$ and $926 \mathrm{~cm}^{-1}$ was explained $\mathrm{C}-\mathrm{H}$ in-plane bending and out-plane bending. The characteristic peaks of PPy layer of composite films (b) were similar to these of pure PPy. However, characteristic absorption peaks of pure CA film (c) were not observed in the spectra of PPy layer of composite films (b). This suggested that the thin and compact layer of the conductive polypyrrole was grown on and adhered tightly to the surface of CA substrates.

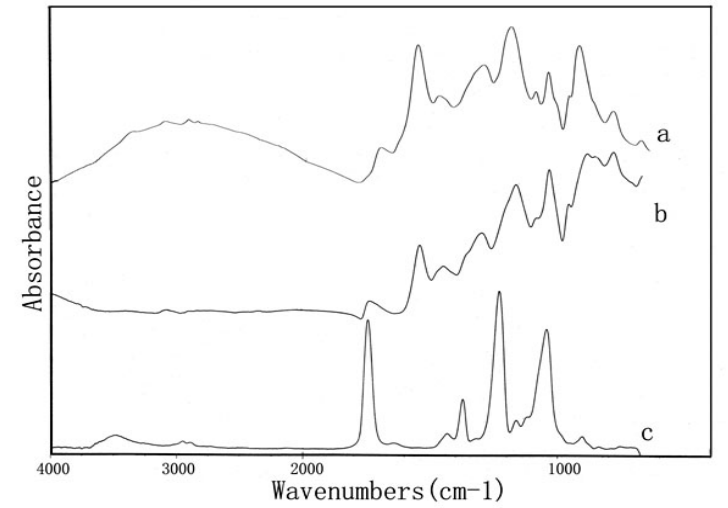

Fig. 2 FTIR spectrum of (a) $\mathrm{FeCl}_{3}$-doped Ppy, (b) polypyrrole/CA films, and (c) CA film.

Under optimum conditions the effect of polymerization temperature on the film resistance was also studied and shown in Fig.3. The film resistance increases evidently with increasing polymerization temperature. Rising temperature is able to increase the reaction rate of polymerization, resulting in formation of oligomers with poor conductance. In order to obtain the composite films with high conductivity, polymerization temperature is controlled at about $10^{\circ} \mathrm{C}$.

The varieties of the film resistance with polymerization time were shown in Fig. 4 . When the other conditions were maintained at the optimum value, the resistance of composite films decreases firstly, and then keeps approximately constant with the increase of polymerization time. Formation of PPy chains with high conductivity needs a suitable reaction time. Short time of oxidation polymerization is unfavorable for long chains of PPy. So the optimum polymerization time is 30 min. 


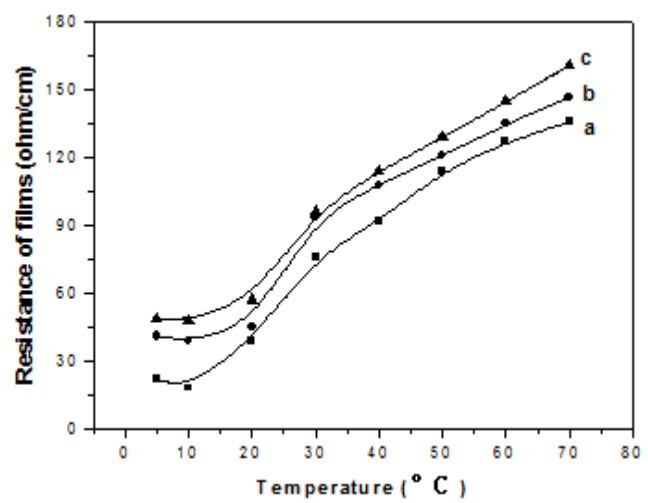

Fig. 3 Temperaturedependence of the films resistance
(a) $1.5 \mathrm{~g}$
(b) $2.0 \mathrm{~g}$
(c) $2.5 \mathrm{~g}$

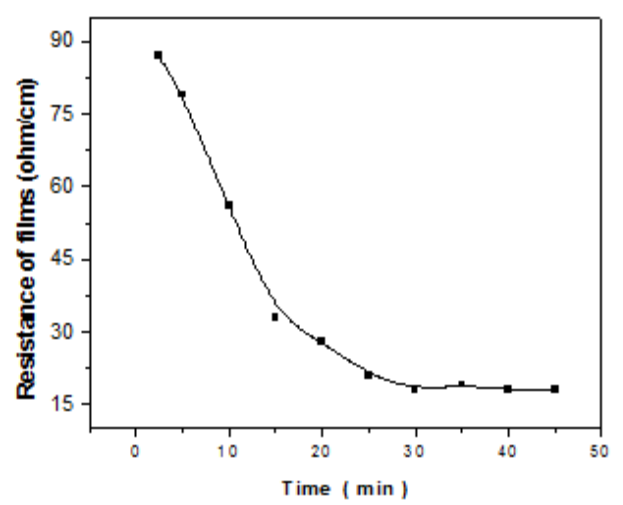

Fig. 4 Time dependence of the films resistance

The thermogravimetric curves for the pure acetate cellulose film and PPy/CA film were shown in Fig. 5a and 5b respectively. It can be seen from Fig.5a that the pure cellulose acetate film is stable up to $120^{\circ} \mathrm{C}$ and completely decomposed around at $500^{\circ} \mathrm{C}$. Fig. 5b demonstrates that $\mathrm{PPy} / \mathrm{CA}$ composite is stable up to $220^{\circ} \mathrm{C}$, the main weight loss occured from 240 to $380^{\circ} \mathrm{C}$.

The weight loss of the PPy/CA film needs higher temperature in comparison with the pure acetate cellulose film. There are two main reasons: Firstly decomposition of PPys is in range of $250-350^{\circ} \mathrm{C}$. Secondly the hydrogen bond formation between PPy and cellulose acetate polymer network and PPy embedded inside the CA matrix reduces moisture absorption in cellulose acetate.
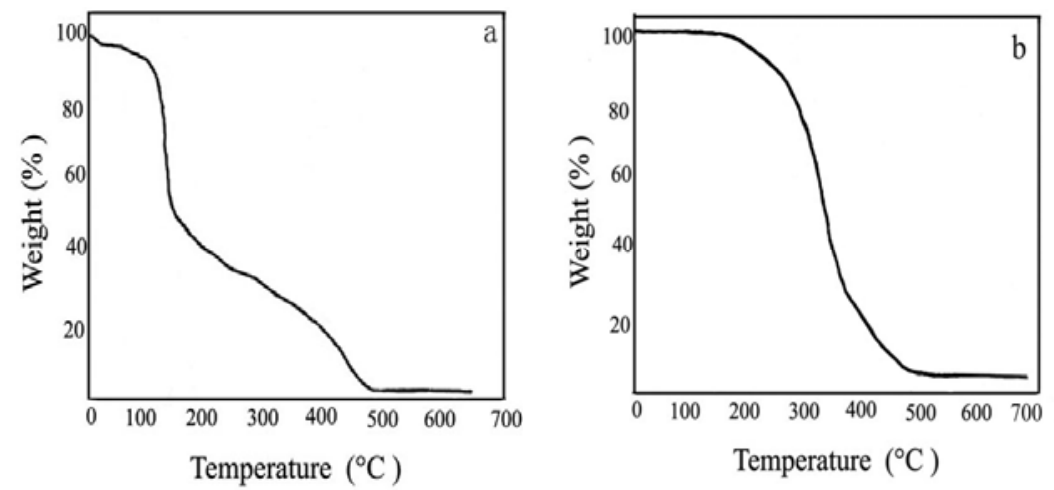

Fig.5. TG analysis curves of (a) pure CA film, and (b) PPy-CA films

\section{Conclusion}

Polypyrrole/cellulose acetate composite films were successfully prepared by phase separation and in-situ polymerization of pyrrole with $\mathrm{FeCl}_{3}$ as oxidant in aqueous medium. The comparison between FTIR spectra of the composite films and pure CA film indicated that the surface of the films was covered with polypyrrole. AFM images of the composites showed that polypyrrole with a cluster-like structure grew on the cellulose acetate matrix. The thermogravimetric analysis indicates that the thermal stability of the PPy/CA film is higher than that of the pure CA film.

\section{Acknowledgments}

This work was supported by Foundation of Gansu Educational Committee (no.2015B-150) and the Lanzhou Petrochemical Vocational and Technical CollegeResearch Fund (no.JY2014-17).

\section{Reference}

[1] J. Chen, A.K. Burrell, G.E. Collis, et al. Electrochim. Acta 47 (2002) 2715. 
[2] J.N. Barisci, G.G. Wallace, M.K. Andrews, et al. Sens. Actuators B 84 (2002) 252.

[3] W. Sun, R. Zheng, X. Chen, J. Power Sources 195 (2010) 7120-7125.

[4]KIM J H , SHARMA A K, L EE Y S. Mater. Lett ., 2006 , 60 (13-14) : 1697-1701.

[5]LIU X L , L YJ , HAN S , et al . Adv. Mater. , 2005. 17 (22) : 2727-2732.

[6]. E. Desimoni, B. Brunetti, P. Ugo. Synthetic Metals 130 (2002) 135-137

[7]. T. Lindfors, J. Bobacka, A. Lewen stam, et al. Electrochim. Acta 43 (1998 )3503.

[8] H. Hammache, L. Makhloufi, B. Saidani. Synth. Met. 123(2001) 515-522

[9] N.V. Bhat, A.P. Gadre, V.A. Bambole. J. Appl. Polym.Sci. 80 (2001) 2511.

[10] YANG X M, DAI T Y, LU Y. Polymer, 2006, 47: 441-447.

[11] Mukoma P, Jooste B R, Vosloo H C M . J . Membr . Sci. , 2004, 243( 1-2) : 293—299.

[12]KOEHL ER S , UEDA M , EFIMOV I , et al . Electrochimica Acta , 2007 , 52 ( 9) :3040-3046. 